\title{
Discussion about Improvement of Stability of the Scan Timing by Placing Small ROI in Cerebral 3D-CTA
}

\author{
Yuichi Watanabe ${ }^{1,2 *}$, Kenji Ino ${ }^{1}$, Kohki Yoshikawa ${ }^{2}$ \\ ${ }^{1}$ Department of Radiological Technology, the University of Tokyo Hospital, Tokyo, Japan \\ ${ }^{2}$ Graduate Division of Health Sciences, Komazawa University, Tokyo, Japan \\ Email: *watanabeyu-rad@h.u-tokyo.ac.jp
}

Received 18 November 2015; accepted 19 December 2015; published 22 December 2015

Copyright (C) 2015 by authors and Scientific Research Publishing Inc.

This work is licensed under the Creative Commons Attribution International License (CC BY). http://creativecommons.org/licenses/by/4.0/

\section{Open Access}

\begin{abstract}
In three-dimensional computed tomography angiography (3D-CTA) in our facility, we usually scan the volume of the brain according to the bolus tracking method. Fluoroscopic slice is placed at the Willis's ring and the timing of scan is determined subjectively by a radiological technologist after strong enhancement of the basal cerebral artery is confirmed. In these procedures, however, variation of scan timing is often problematic. Therefore, we design the surpassing method to place the small region-of-interest (ROI) at the basal cerebral arteries and to start CT scan automatically. In this protocol, the fluoroscopic slices of the distal internal carotid arteries are selected referring to the precontrast volume data, small ROIs are set in bilateral internal carotid arteries, and scan trigger of CT is started automatically at the threshold of $170 \mathrm{HU}$. The maximum $80 \mathrm{~mL}$ of iodine contrast agent $300 \mathrm{mgI} / \mathrm{mL}$ is injected intravenously at the rate of $4.0 \mathrm{~mL} / \mathrm{sec}$, and the volume of the arterial phase is scanned automatically. We measure ROIs at the internal carotid arteries based on the obtained volume data of arterial phase and estimate the optimal scan timings from the fluoroscopic CT images reformatted at the intervals of $0.1 \mathrm{sec}$. In 38 of 53 patients, placement of the small ROIs is succeeded and automatic or manual CT scan is performed. In the patients who succeed in placement of the small ROIs, optimal scan timing of the arterial phase is obtained, while in the patients who fail placement of the small ROIs, a large variation is observed in their scan timings. Their results suggest that more stable scanning of the arterial phase is available by means of small ROI placement and automatic scanning. The clinical significance is large because the stability and reproducibility of the examination provide a quantitative analysis and more accurate diagnosis.
\end{abstract}

\section{Keywords}

Cerebral 3D-CTA, Small ROI, Bolus Tracking, Volume Scan, 320-Row CT

\footnotetext{
${ }^{*}$ Corresponding author.
}

How to cite this paper: Watanabe, Y., Ino, K. and Yoshikawa, K. (2015) Discussion about Improvement of Stability of the Scan Timing by Placing Small ROI in Cerebral 3D-CTA. Open Journal of Radiology, 5, 224-234. 


\section{Introduction}

Three-dimensional computed tomography angiography (3D-CTA) of the brain is performed for 3D morphological analysis of the aneurysm or arteriovenous malformation and their postoperative follow-up. In 3D-CTA, multi-detector CT (MDCT) is used [1]-[4]. 3D-CTA generally consists of the precontrast helical scan to be used as the data for subtraction and arterial phase helical scan that is performed after potent enhancement of the interest artery is confirmed in the fluoroscopic slice at the neck [5] [6]. Recently, multi-detector CT has been developed markedly. In 320-row MDCT scanner with $320 \times 0.5 \mathrm{~mm}$ detectors (TOSHIBA Aquilion ONE, Toshiba Medical Systems Corp, Japan) covers scanning of the whole brain in one 16-cm gantry rotation, and is able to use for the volume scan [7]-[12]. In our University of Tokyo Hospital, 3D-CTA of the brain has also been performed for volume scanning with 320-row MDCT by bolus tracking method [13] [14]. The stability and reproducibility of the examination greatly depend on the skill of a radiological technologist, because scan timing for obtaining arterial phase is visually determined by a radiological technologist based on the enhancement of the interest artery. As a result, variation of the scan timing for the arterial phase becomes problematic in our hospital. Too early start of scanning results in poor image quality of arterial phase, and delayed start need selective removal of the enhanced vein image due to high CT values of the vein, which increases burden to the radiological technologist who prepares the reconstructed VR or MIP images.

\section{Purposes}

This study is aimed to improve the reliability of time-density curve (TDC) by placing the small region-of-interest (ROI) in the intracranial artery on the fluoroscopic image and to optimize scan timing at specified threshold [15]. We describe the routine procedures of cerebral 3D-CTA in our hospital. The results are compared among the patients who succeeded and failed placement of the small ROI and a relationship between the accuracy of the small ROI placement and the timing of scan start is verified from TDCs.

\section{Used Equipment}

All examinations in this study were performed with 320-row MDCT scanner (TOSHIBA Aquilion ONE, Toshiba Medical Systems Corp, Japan). Aquilion ONE has $320 \times 0.5 \mathrm{~mm}$ detectors and covers the width of $16 \mathrm{~cm}$ in one rotation. We used ZioStation 2 (ZiosoftInc, Japan), which is a medical image processing workstation, to process the VR images. And we used ImageJ v1.45 for ROI analysis and drawing TDC.

\section{Subjects and Methods}

\subsection{Subjects}

The subjects in this study were 53 patients (14 male, 39 female; 24 - 85 years, mean 58 years) those had cerebral 3D-CTA volume scan during the period from March 30 to June 20 in 2012. We scanned the range of $16 \mathrm{~cm}$ in one rotation in this protocol. So if the purpose of examination could be achieved in that range of scan, the patients were included in this study. If the range of interest was more than $16 \mathrm{~cm}$, examination by helical scan was selected. The purposes of each examination and positioning of the fluoroscopic slice are described in Table 1. Written informed consent was obtained from all patients, and the study protocol was approved by the institutional ethics committee.

\subsection{Cerebral 3D-CTA Volume Scan Protocol}

The protocol of cerebral 3D-CTA volume scan in our hospital is summarized in Figure 1. In the cerebral 3DCTA, volume scan was performed by bolus tracking method with 320-row MDCT. The dose of $80 \mathrm{~mL}$ of iodine contrast agent $300 \mathrm{mgI} / \mathrm{mL}$ was injected at a rate of $4.0 \mathrm{~mL} / \mathrm{sec}$.

The characteristics of our study were to determine the position of fluoroscopic slice which includes the distal internal carotid arteries if significant artifacts due to calcification or surgical clip are not observed in the slice, to determine the optimal scan timing in CT fluoroscopy, and to perform once scan for the whole brain. The advantage of volume scanning is able to scan whole vasculatures simultaneously, whereas in conventional caudalcranial helical scanning different vascular phases are scanned at the starting and end points. Moreover, placement 
Table 1. Purpose of each examination and positioning of the fluoroscopic slice.

\begin{tabular}{ccc}
\hline Purpose of the examination & Number of cases \\
\hline Aneurysm & Preoperation & 20 \\
& Postoperation & 16 \\
\hline Dissection, stenosis, occlusion and hemorrhage & 7 \\
Evaluation of vessels before surgery & Number of cases \\
\hline Position of the fluoroscopic slice & 3 \\
\hline External acoustic foramen & 47 \\
\hline Distal internal carotid artery & 3 \\
\hline
\end{tabular}

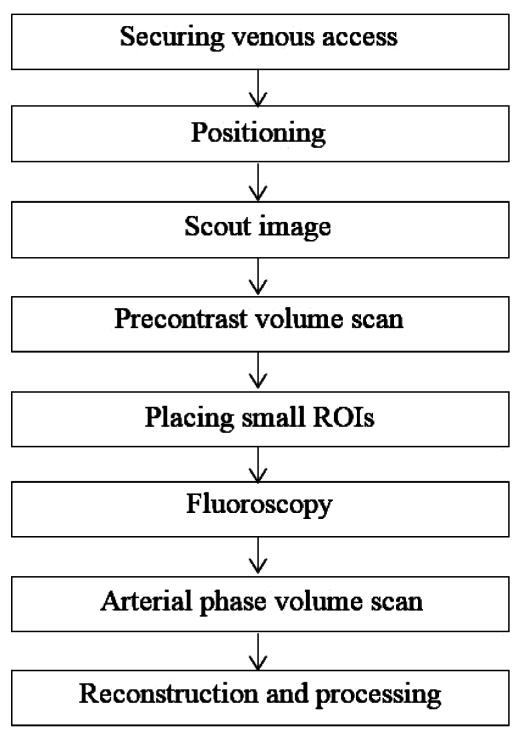

Secure median vein of right elbow.

Adjust to OM line, fix the head and warn carefully not to move.

Take 2 scout images of 0 and 90 degrees.

FOV $220,120 \mathrm{kV}, 200 \mathrm{~mA}, 1.0 \mathrm{sec}, 0.5 \mathrm{~mm}$, FC64, AIDR Mild, small focus.

Place the small ROIs at the distal internal arteries.

FOV $220,120 \mathrm{kV}, 50 \mathrm{~mA}, 1.0 \mathrm{sec}, 2.0 \mathrm{~mm}$

FOV $220,120 \mathrm{kV}, 270 \mathrm{~mA}, 1.0 \mathrm{sec}, 0.5 \mathrm{~mm}$, FC64, AIDR Mild, small focus.

FOV 130, thickness 0.5 / interval $0.25 \mathrm{~mm}$, FC43, AIDR Mild.

Process multi-planar reconstruction, volume rendering (subtraction $+/-$ ) and maximum intensity projection images.

\section{Figure 1. Summary of cerebral 3D-CTA volume scan protocol.}

of the fluoroscopic slice at the distal internal carotid artery near the center of the cranial cavity can save the time from triggering of scan start and to secure the arterial phase of the interest region that is the favorite site of cerebral aneurysm.

However, there are some problems. First, exact scan timing is required because of once volume scanning. Second, the stability and reproducibility of the examination highly depend on the ability of a radiological technologist because scan timing is visually determined by a radiological technologist based on enhancement of the interest artery.

Therefore, we introduced the methods to place the small ROIs of cerebral arteries in the fluoroscopic image, to draw TDCs, and allocate the thresholds to the CT numbers in the small ROI in order to determine the timing of scan start, not the visual determination by a radiological technologist. The distal internal cerebral artery is selected as the fluoroscopic interest region in general. This position is easy to secure the internal carotid artery extended perpendicular to the slice and hard to be affected by the partial volume effect of the adjacent bone, because there are less bony systems around there. The small ROIs were placed bilaterally with the size to be contained in each internal carotid artery. CT values at the small ROI were determined at the threshold of $170 \mathrm{HU}$ and the arterial phase image was obtained. Outlines and layouts of the small ROI at the distal internal carotid artery are shown in Figure 2 and Figure 3, respectively.

In addition, if there are metal artifacts due to postsurgical arterial clips or coils on the slice in the distal internal 


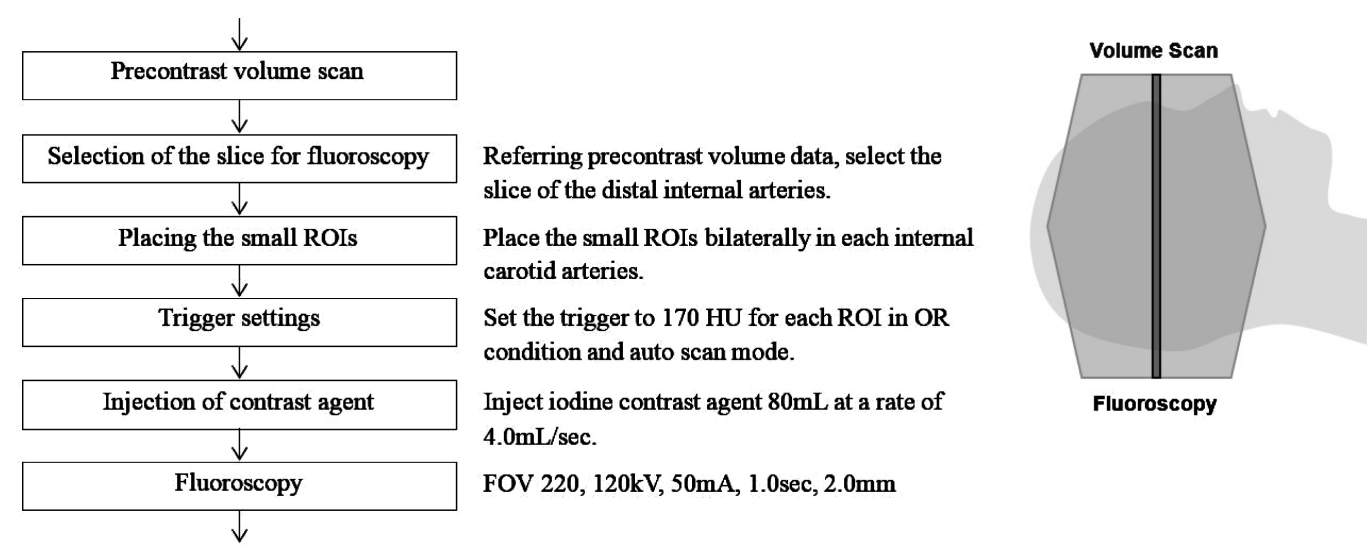

Figure 2. Outlines of placement of the small ROIs at the distal internal carotid arteries.

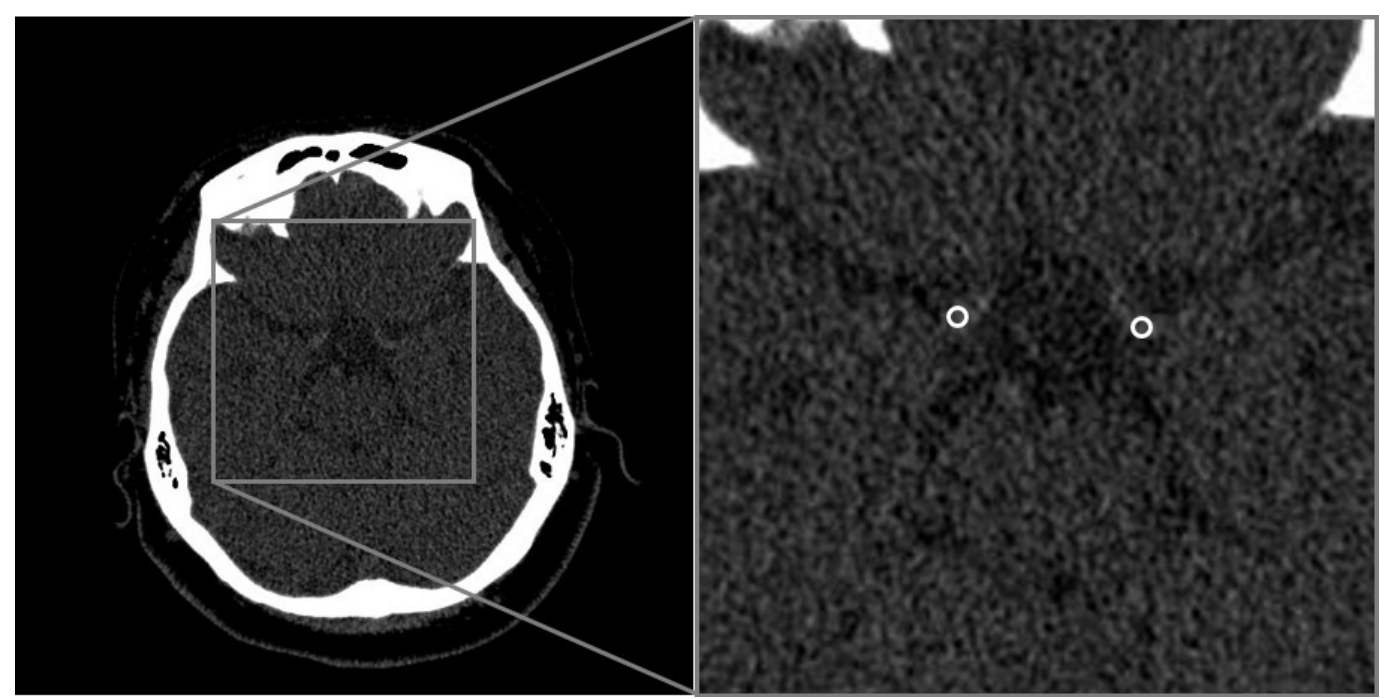

Figure 3. Example of layout of the small ROIs at the distal internal carotid arteries.

carotid artery, the fluoroscopic slice should be selected more caudally in order to avoid influences of the artifact. The small ROI was placed within each internal carotid artery. Even with faint movement of a patient, bony structure will be contained in the small ROI and the trigger to start will be induced before the internal arterial enhancement is observed. Therefore, we started manual scan at the threshold of 170 HU referencing to the TDC obtained. Outlines and layouts of the small ROI that were placed more caudally to the distal internal carotid artery are shown in Figure 4 and Figure 5, respectively.

The mean CTDIvol and DLP were $285 \mathrm{mGy}$ and $1530 \mathrm{mGy} \cdot \mathrm{cm}$ in this protocol.

\subsection{Relationship between Accuracy of the Small ROI Placement and Timing of the Scan Start}

The fluoroscopic images were reconstructed with FC64, Full, and $220 \mathrm{~mm}$ FOV at the intervals of 0.1 sec, and TDCs were drawn at the small ROIs that were placed at the most ideal position within the internal carotid arteries. The relationship between accuracy of the small ROI placement and timing of the scan start were verified.

\subsection{Evaluation of Scan Timing}

In order to evaluate scan timing of the arterial phase based on the volume data reconstructed with FC64, full, 0.5 mm slice thickness, and $220 \mathrm{~mm}$ FOV, the small ROI was placed at the distal bilateral internal carotid arteries for measurement of the arterial CT values and at the heads of sinus rectus and bilateral sigmoid venous sinus 


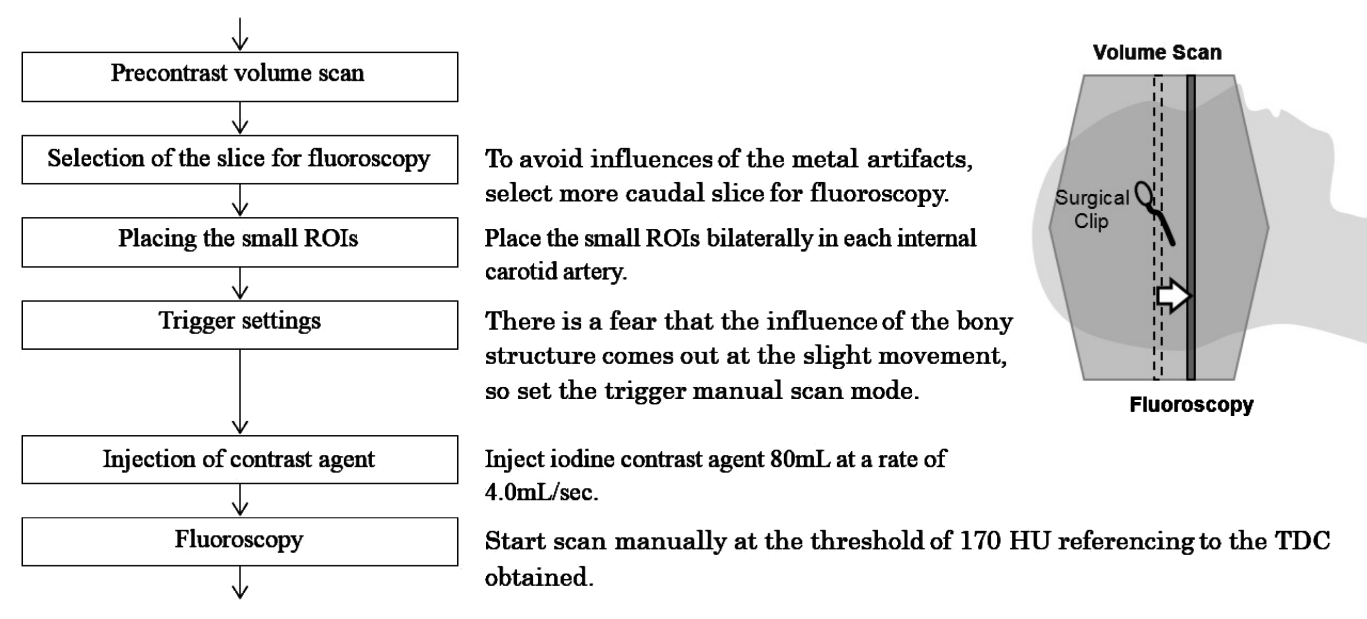

Figure 4. Outlines of placement of the small ROI if more caudal slice is selected for the fluoroscopic slice.

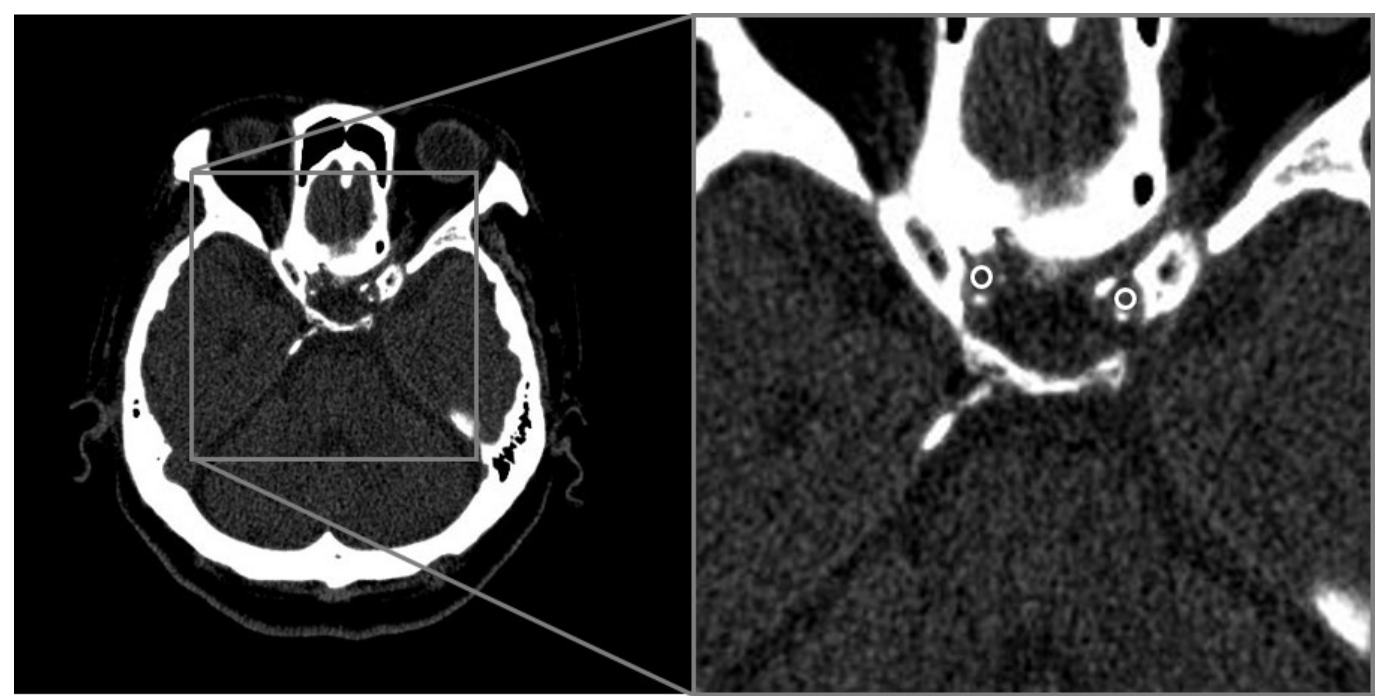

Figure 5. Example of layout of the small ROIs if more caudal slice is selected for fluoroscopy.

for measurement of venous CT values, respectively. Software of ImageJ (version 1.45) was used for calculation of these CT values.

\section{Results}

\subsection{Relationship in TDC between Small ROI Placement and Scan Timing}

The volume rendering (VR) images (WL/SH=100/79) in which the external carotid arteries and their branches and the artifacts introduced by misregistration were removed from the images, the small ROIs actually placed for the examination, the small ROIs placed for analysis and their TDCs are detailed below for 4 cases.

\subsubsection{Case 1}

A 44-year-old female was suspected craniopharyngioma and had 3D-CTA for preoperative examination of intracranial vessels, shown in Figure 6. The fluoroscopic slice was selected at the distal internal carotid artery according to the study protocol. The small ROIs were placed within the bilateral internal carotid arteries, and 3DCTA was started automatically at the threshold of $170 \mathrm{HU} 16.6 \mathrm{sec}$ after intravenous injection of contrast agent. The reproducibility of TDC at the small ROI placed after examination was satisfactory, and the CT values of the small ROI increased at $170 \mathrm{HU} 13.3 \mathrm{sec}$ after the contrast agent was injected. The CT values of the internal 


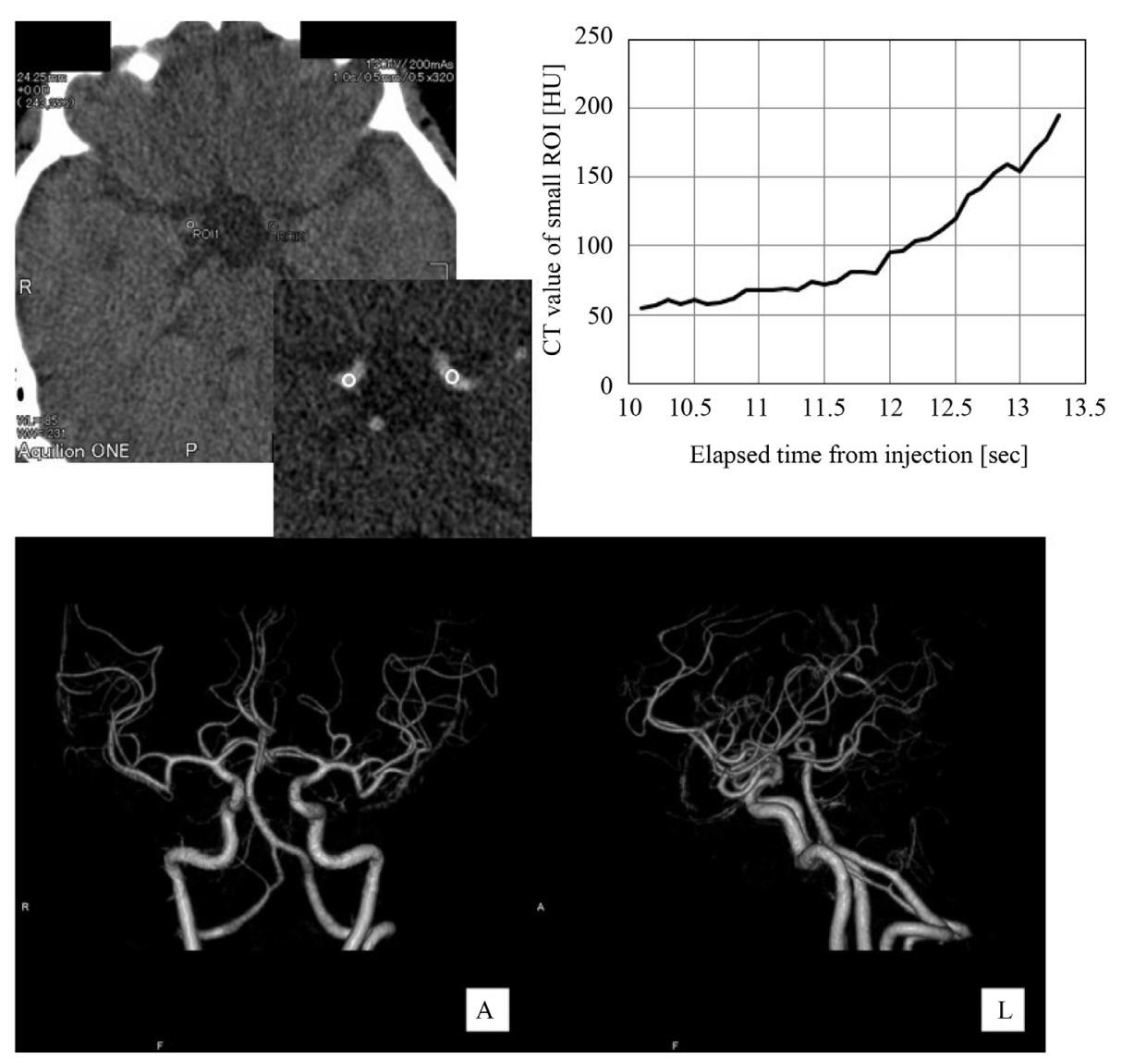

Figure 6. The small ROIs, TDC and VR images in case 1.

carotid artery and the straight sinus were $312 \mathrm{HU}$ and $77 \mathrm{HU}$, respectively, which suggests that the arterial system alone was enhanced at this timing. In the VR images, strongly enhanced peripheral arteries were drawn clearly and the venous system was little observed. It was suggested that the small ROIs were placed accurately.

\subsubsection{Case 2}

A 59-year-old female had 3D-CTA for postoperative follow-up examination, shown in Figure 7. She had clipping surgery to the aneurysm of the left posterior inferior cerebellar artery 8 years ago. The fluoroscopic slice was selected at the distal internal carotid artery. The small ROIs were placed within the bilateral internal carotid arteries, and 3D-CTA was started automatically at the threshold of $170 \mathrm{HU} 16.1 \mathrm{sec}$ after intravenous injection of contrast agent. The reproducibility of TDC at the small ROI placed after examination was satisfactory, and CT values of the small ROI increased at $170 \mathrm{HU} 12.7 \mathrm{sec}$ after the contrast agent injected. The CT values of the internal carotid artery and the straight sinus were $336 \mathrm{HU}$ and $87 \mathrm{HU}$, respectively. In VR image, arterial system alone was selectively enhanced, which suggests that the small ROIswere placed accurately.

\subsubsection{Case 3}

A 55-year-old female had 3D-CTA for postoperative follow-up examination, shown in Figure 8. She had clipping surgery to the aneurysm of the left posterior inferior cerebellar artery 20 years ago. More caudal slice was selected for fluoroscopy, because clip-related strong metal artifact was observed at the internal carotid artery, and was unable to select the distal internal carotid artery for fluoroscopic slice. There was bony system near the internal carotid artery, which is a potential risk for the bone to be included in the small ROI. Therefore, scanning was started manually at the threshold of $170 \mathrm{HU}$ referencing to TDC $19 \mathrm{sec}$ after intravenous injection of the contrast agent. The reproducibility of TDC at the small ROI that was placed after examination was satisfactory, 

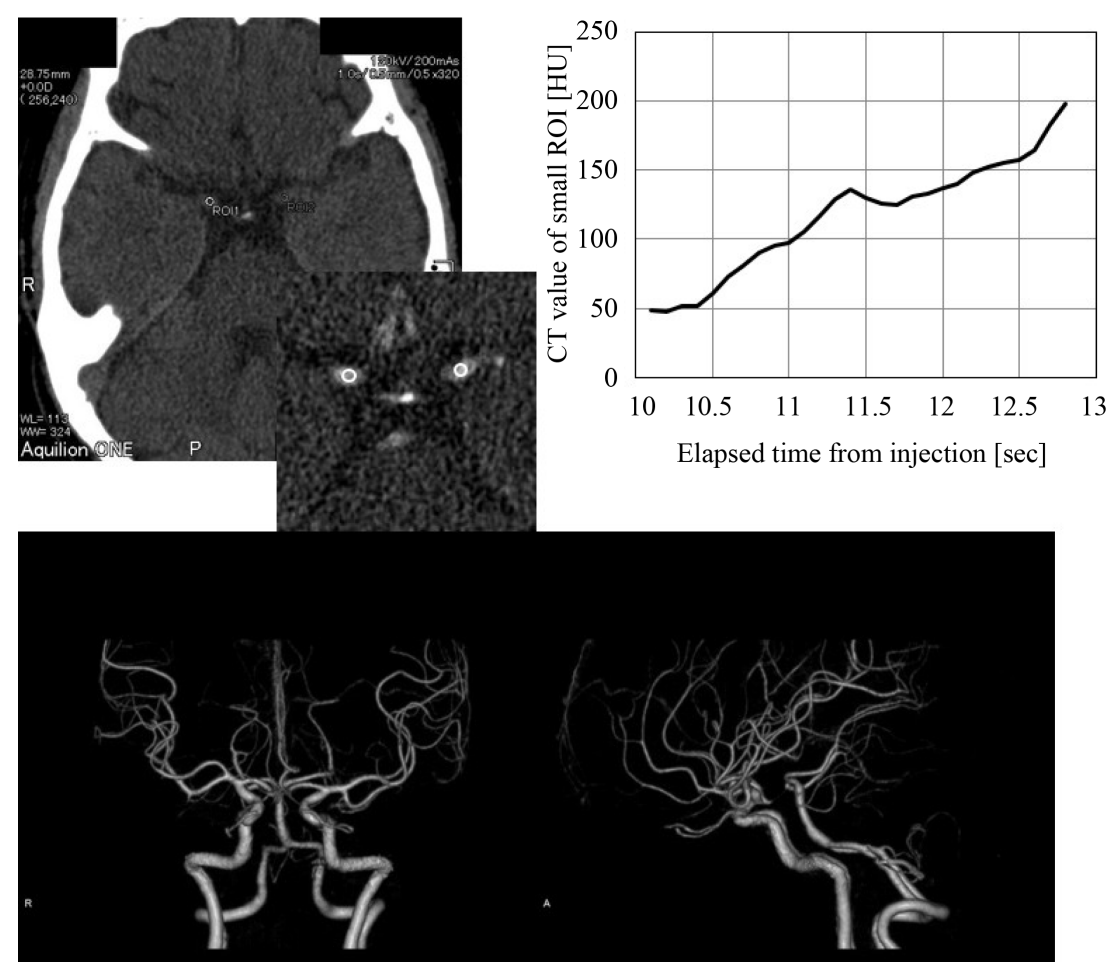

A

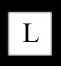

Figure 7. The small ROIs, TDC and VR images in case 2.
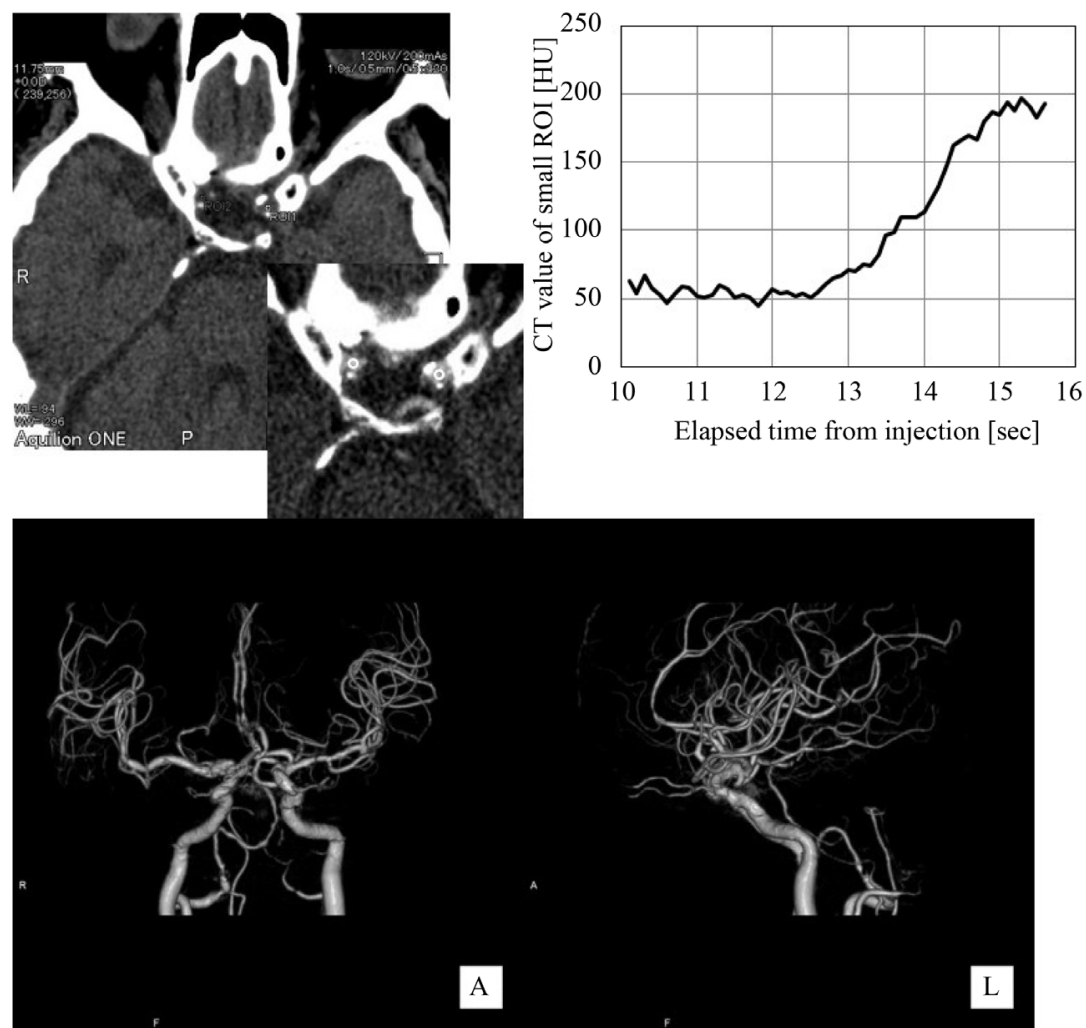

Figure 8. The small ROIs, TDC and VR images in case 3. 
and the CT values of the small ROI increased at $170 \mathrm{HU} 15$ sec after intravenous injection of the contrast agent. The CT values of the internal carotid artery and the straight sinus were $465 \mathrm{HU}$ and $110 \mathrm{HU}$, respectively. In VR image, arterial system alone was selectively enhanced, which suggests that the small ROI was placed accurately. As seen in this case, timing of scan is optimized by placement of the small ROI, even if fluoroscopic slice is not located at the distal internal carotid artery. However, neighboring bony system is included in the small ROI by faint movement of a patient. As a result, the mean CT value of ROI increases, and scanning is started before the contrast agent reaches at the distal internal carotid artery. In such a case, scanning should be manually started referencing to the TDC.

\subsubsection{Case 4}

A 60-year-old female had 3D-CTA for postoperative follow-up examination, shown in Figure 9. She had clipping surgery to the aneurysm at the left internal carotid artery-posterior communicating artery 7 days ago. The fluoroscopic slice was selected at the distal internal carotid artery. The small ROIs were placed within the bilateral internal carotid arteries, and 3D-CTA was started automatically at the threshold of $170 \mathrm{HU} 17.3 \mathrm{sec}$ after intravenous injection of contrast agent. For the TDC placed after examination, the CT values of the small ROI increased at $270 \mathrm{HU} 14 \mathrm{sec}$ after the contrast agent was injected. The CT values of the internal carotid artery and the straight sinus were $374 \mathrm{HU}$ and $175 \mathrm{HU}$, respectively, which suggests that timing of the scan is slightly delayed. In VR images, the venous system was clearly drawn. This delay of scan timing is related to the decreased mean CT values in the small ROI because the extra-arterial component was included in the small ROI placed during examination.

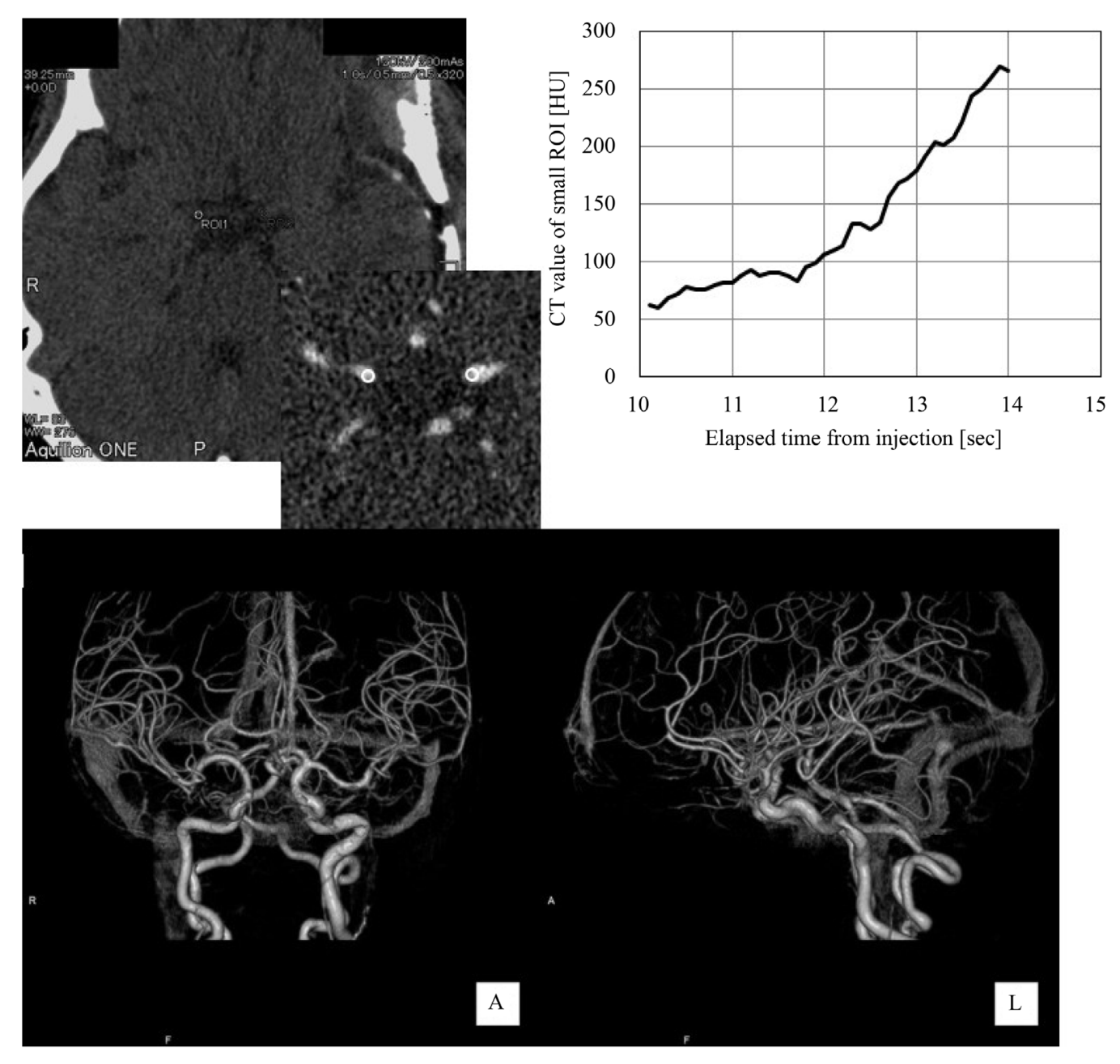

Figure 9. The small ROIs, TDC and VR images in case 4. 


\subsection{Success Rate of the Small ROI Placement}

Out of the 53 patients examined, 37 patients were scanned automatically and 16 patients manually. The results of automatic/manual scanning, success/failure of ROI placement, and timing of scan are shown in Table 2.

The ROI was placed satisfactory in 33 of 37 patients scanned automatically. Most of these 33 patients were scanned at optimal timing though a little early timing of scan start was observed in five of them. In remaining four cases, the CT values of the venous system were slightly higher and the timings of scan start were slightly delayed. In five of 16 patients manually scanned, the ROI was placed satisfactory and scanning was started at optimal timing with highly reliable TDC.

In remaining 11 patients, the conventional visual arterial scanning was performed because the small ROI was mislocated, the fluoroscopic positions were inappropriate and the patients moved during the period of time from placement of the small ROI to start of fluoroscopy. Of the 11 patients performed visual scanning, the timing of scan start was earlier in two, adequate in seven and delayed in two patients, respectively.

Overall, placement of the small ROI was successful and the arterial phase was scanned satisfactory in 38 (71.7\%) of 53 patients, while placement of the small ROI was failed and timing of the scan was varied in 15 patients (28.3\%).

\subsection{Evaluation of Scan Timing}

The results of automatic/manual scan start, success/failure placement of the small ROI, and timing of scan in 53 patients are shown in Table 2, and the scatter diagram plotted arterial and venous CT values is shown in Figure $\mathbf{1 0 .}$

If the contrast between arterial and venous systems is higher and the CT value of the venous system is lower, more exact arterial phase is obtained and the examination becomes more meaningful. The condition of CT values less than $150 \mathrm{HU}$ for venous system and more than $250 \mathrm{HU}$ for arterial system saves the time to reconstruct VR images and produce satisfied results. As shown in Figure 10, CT values are less than 150 HU and homogeneously distributed when placement of the small ROI succeeded $(\bullet)$. The arterial phase was scanned at optimal scan timing in most of these patients, though the scan timing in 5 of them was slightly earlier. On the contrary, in the patients who failed placement of the small ROI $(\times)$, large variation was observed in distribution of CT values.

Table 2. Success/failure of placement of the small ROIs and scan timing.

\begin{tabular}{|c|c|c|c|c|c|}
\hline Scan start & Number of cases & ROI placement & Number of cases & Scan timing & Number of cases \\
\hline \multirow[t]{2}{*}{ Auto } & 37 & Accurate & 33 & Proper & 33 \\
\hline & & Mislocated & 4 & Delay & 4 \\
\hline \multirow[t]{4}{*}{ Manual } & 16 & Accurate & 5 & Proper & 5 \\
\hline & & Mislocated & 11 & Early & 2 \\
\hline & & & & Proper & 7 \\
\hline & & & & Delay & 2 \\
\hline
\end{tabular}

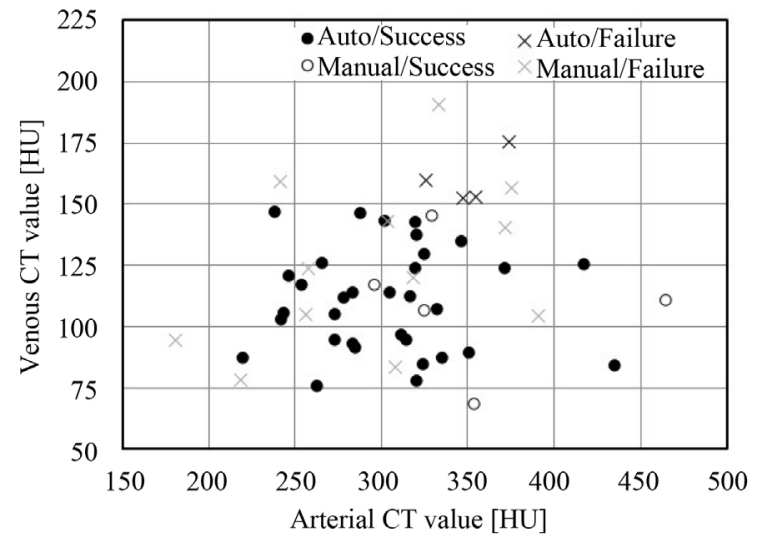

Figure 10. Distribution of arterial/venous CT value. 


\section{Discussions}

Though there have been several reports on coronary artery CT by bolus tracking method with 320-row MDCT [16] [17], there is no report on cerebral 3D-CTA. Cerebral helical CTA by bolus tracking in thoracic aorta has been reported [18], but that in intracranial artery using 320-row MDCT has not been reported.

In the study protocol, fluoroscopic slice is placed at the distal internal carotid artery. Selection of the distal internal carotid artery for fluoroscopic slice is considered to be ideal from the four advantages. First, the time from triggering of the scan start to actual scanning of the arterial phase is short because the small ROI is near the center of the volume scan. Second, the target arterial phase is directory secured because the position of the small ROI is consistent with the favorite site of cerebral aneurysms. Third, there is little partial volume effect along the body axis, because the internal carotid artery is extended perpendicular to the fluoroscopic slice. Finally, a risk of bony involvement in ROI slice is very low, because there are little bones peripherally other than the dorsum sella.

In all 38 patients succeeded in placing small ROI, the CTA was performed at the acceptable timing. The key point for successful scanning is placement of the small ROI. If placement of the small ROI is succeeded, reliability of TDC improves significantly and stability of the study is secured. In 5 patients of them, scanning was started at slightly early timing. The need to increase the threshold for scan-starting was suggested in the examination that bolus characteristics of contrast agent is secured because the scan parameters for contrast enhancement were fixed regardless of physical build of a patient. In addition, scanning parameters should be reviewed in detail including fixed iodine dose per kg body weight in the future.

With the threshold of $170 \mathrm{HU}$, automatic scanning was started at the optimal scan timing in 33 (87\%) of 38 patients, and these thresholds seem to be reasonable. In four patients, placement of small ROI appeared to be succeeded, but scan timing was slightly delayed because extra-arterial component was included in the small ROI and the mean CT values decreased. The CT values of artery and vein were 320 - $370 \mathrm{HU}$ and 150 - $175 \mathrm{HU}$, respectively, and they were within acceptable limits in all these 4 patients, but careful attention should be paid to the positional relationship between the small ROI and the artery during fluoroscopy. If the extra-arterial component is included in the small ROI, TDC values is lower than the values observed when the small ROI is properly placed, and manual scan start should be considered.

\section{Conclusion}

The key point for stable scanning of the arterial phase is the positioning of the small ROI in cerebral 3D-CTA. Placement of the small ROI is succeeded in 38 of 53 patients, and scanning is started at the optimal timing. Stability of the examination is improved by optimal placement of the small ROI. The clinical significance is large because the stability and reproducibility of the examination provide a quantitative analysis and more accurate diagnosis.

\section{References}

[1] Tanabe, S., Ohtaki, M., Uede, T., Hashi, K., Suzuki, S. and Takahashi, H. (1995) Diagnosis of Ruptured and Unruptured Cerebral Aneurysms with Three-Dimensional CT Angiography (3D-CTA). Neurological Surgery, 23, 787-795.

[2] Gonzalez-Darder, J.M., Pesudo-Martinez, J.V. and Feliu-Tatay, R.A. (2001) Microsurgical Management of Cerebral Aneurysms Based in CT Angiography with Three-Dimensional Reconstruction (3D-CTA) and without Preoperative Cerebral Angiography. Acta Neurochirurgica, 143, 673-679. http://dx.doi.org/10.1007/s007010170045

[3] Inoue, S., Hosoda, K., Fujita, A., Ohno, Y., Fujii, M. and Kohmura, E. (2011) Diagnostic Imaging of Cerebrovascular disease on Multi-Detector Row Computed Tomography (MDCT). Neurological Surgery, 63, 923-932.

[4] Kato, Y., Nair, S., Sano, H., Sanjaykumar, M.S., Katada, K., Hayakawa, M. and Kanno, T. (2002) Multi-Slice 3D-CTA —An Improvement over Single Slice Helical CTA for Cerebral Aneurysms. Acta Neurochirurgica, 144, 715-722. http://dx.doi.org/10.1007/s00701-002-0932-7

[5] Jayakrishnan, V.K., White, P.M., Aitken, D., Crane, P., McMahon, A.D. and Teasdale, E.M. (2003) Subtraction Helical CT Angiography of Intra- and Extracranial Vessels: Technical Considerations and Preliminary Experience. American Journal of Neuroradiology, 24, 451-455.

[6] Watanabe, Y., Uotani, K., Nakazawa, T., Higashi, M., Yamada, N., Hori, Y., Kanzaki, S., Fukuda, T., Itoh, T. and Naito, H. (2009) Dual-Energy Direct Bone Removal CT Angiography for Evaluation of Intracranial Aneurysm or Stenosis: Comparison with Conventional Digital Subtraction Angiography. European Radiology, 19, 1019-1024. 
http://dx.doi.org/10.1007/s00330-008-1213-5

[7] Kumamaru, K.K., Hoppel, B.E., Mather, R.T. and Rybicki, F.J. (2010) CT Angiography: Current Technology and Clinical Use. Radiologic Clinics of North America, 48, 213-235. http://dx.doi.org/10.1016/j.rcl.2010.02.006

[8] Hayakawa, M., Maeda, S., Sadato, A., Tanaka, T., Kaito, T., Hattori, N., Ganaha, T., Moriya, S., Katada, K., Murayama, K., Kato, Y. and Hirose, Y. (2011) Detection of Pulsation in Ruptured and Unrupturedcerebral Aneurysms by Electrocardiographically Gated 3-Dimensional Computed Tomographic Angiography with a 320-Row Area Detector Computed Tomography and Evaluation of Its Clinical Usefulness. Neurosurgery, 69, 843-851. http://dx.doi.org/10.1227/NEU.0b013e318225b2d3

[9] Siebert, E., Bohner, G., Masuhr, F., Deuschle, K., Diekmann, S., Wiener, E., Bauknecht, H.-C. and Klingebiel, R. (2010) Neuroimaging by 320-Row CT: Is There a Diagnostic Benefitor Is It Just Another Scanner? A Retrospective Evaluation of 60 Consecutive Acute Neurological Patients. Neurological Sciences, 31, 585-593. http://dx.doi.org/10.1007/s10072-010-0292-7

[10] Tomizawa, N., Nojo, T., Akahane, M., Torigoe, R., Kiryu, S. and Ohtomo, K. (2012) Adaptive Iterative Dose Reduction in Coronary CT Angiography Using 320-Row CT: Assessment of Radiation Dose Reduction and Image Quality. Journal of Cardiovascular Computed Tomography, 6, 318-324.

[11] Sun, Z.H., Al Moudi, M. and Cao, Y. (2014) CT Angiography in the Diagnosis of Cardiovascular Disease: A Transformation in Cardiovascular CT Practice. Quantitative Imaging in Medicine and Surgery, 4, 376-396.

[12] Motosugi, U., Ichikawa, T., Sou, H., Morisaka, H., Sano, K. and Araki, T. (2012) Multi-Organ Perfusion CT in the Abdomen Using a 320-Detector Row CT Scanner: Preliminary Results of Perfusion Changes in the Liver, Spleen, and Pancreas of Cirrhotic Patients. European Journal of Radiology, 81, 2533-2537. http://dx.doi.org/10.1016/j.ejrad.2011.11.054

[13] Cademartiri, F., van der Lugt A., Luccichenti, G., Pavone, P. and Krestin, G.P. (2002) Parameters Affecting Bolus Geometry in CTA: A Review. Journal of Computer Assisted Tomography, 26, 598-607.

[14] Kirchner, J., Kickuth, R., Laufer, U., Noack, M. and Liermann, D. (2000) Optimized Enhancement in Helical CT: Experiences with a Real-Time Bolus Tracking System in 628 Patients. Clinical Radiology, 55, 368-373. http://dx.doi.org/10.1053/crad.2000.0376

[15] Tenjin, H., Asakura, F., Nakahara, Y., Matsumoto, K., Matsuo, T., Urano, F. and Ueda, S. (1998) Evaluation of Intraaneurysmal Blood Velocity by Time-Density Curve Analysis and Digital Subtraction Angiography. American Journal of Neuroradiology, 19, 1303-1307.

[16] Cademartiri, F., Nieman, K., van der Lugt, A., Raaijmakers, R.H., Mollet, N., Pattynama. P.M.T., de Feyter, P.J. and Krestin, G.P. (2004) Intravenous Contrast Material Administration at 16-Detector Row Helical CT Coronary Angiography: Test Bolus versus Bolus-Tracking Technique. Radiology, 233, 817-823. http://dx.doi.org/10.1148/radiol.2333030668

[17] Stenzel, F., Rief, M., Zimmermann, E., Greupner, J., Richter, F. and Dewey, M. (2014) Contrast Agent Bolus Tracking with a Fixed Threshold or a Manual Fast Start for Coronary CT Angiography. European Radiology, 24, 1229-1238. http://dx.doi.org/10.1007/s00330-014-3148-3

[18] Huang, R.Y., Chai, B.B. and Lee, T.C. (2013) Effect of Region-of-Interest Placement in Bolus Tracking Cerebral Computed Tomography Angiography. Neuroradiology, 55, 1183-1188. http://dx.doi.org/10.1007/s00234-013-1228-8 